
ASCÓRBICO EM SORO DE QUEIJO E SORO DE RICOTA

\title{
ELECTROCHEMICAL EVALUATION OF THE STABILITY OF ASCORBIC ACID IN WHEY FROM CHEESE AND RICOTTA
}

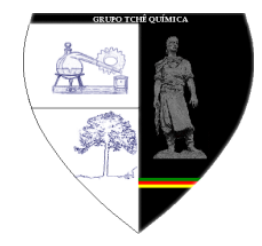

HOEHNE, Lucélia ${ }^{1 *}$; GENNARI, Adriano²; ETHUR, Eduardo Miranda3; HILGEMANN, Maurício*; SOUZA, Claucia Fernanda Volken ${ }^{5}$; SPRANDEL, C. L. ${ }^{6}$; SCHLABITZ, Cláudia7; KUHN, Daniel ${ }^{8}$; ALTMAYER, Taciélen ${ }^{9}$; GONÇALVES, Tamara Engelmann ${ }^{10}$

1,2,3,5,7,8,9,10 Programa de Pós-Graduação em Biotecnologia, Centro Universitário UNIVATES, 95900-000, Lajeado - RS, Brasil.

(fone: +55 $513714-7000$ )

${ }^{3,4,6}$ Programa de Pós-Graduação em Ambiente e Desenvolvimento, Centro Universitário UNIVATES, 95900000, Lajeado - RS, Brasil. (fone: +55 51 3714-7000)

\author{
${ }^{*}$ Autor correspondente \\ e-mail: Iuceliah@univates.br
}

Received 09 August 2016; received in revised form 17 October 2016; accepted 29 November 2016

\section{RESUMO}

O ácido ascórbico tem despertado grande interesse em função da sua capacidade antioxidante, que pode prevenir reações oxidativas. A voltametria cíclica se destaca como uma das principais técnicas eletroquímicas para avaliação de compostos antioxidantes. O soro de queijo e o soro de ricota são subprodutos dos laticínios, e apresentam diversos componentes que permitem sua aplicação em diversos alimentos. $O$ enriquecimento desses subprodutos com substâncias essenciais na alimentação humana podem tornar seu uso ainda mais atrativo pelas indústrias. Portanto, o objetivo deste trabalho foi avaliar a estabilidade do ácido ascórbico em soro de queijo e soro de ricota utilizando voltametria cíclica. Para isso, os soros de queijo e de ricota coletados de uma indústria de laticínios do Vale do Taquari-RS foram enriquecidos com ácido ascórbico e sua estabilidade foi avaliada através do perfil eletroquímico das amostras. Com os dados obtidos, concluiu-se que um dos fatores que afetaram de forma direta na estabilidade do ácido ascórbico foi o $\mathrm{pH}$, porém, mais estudos são necessários para avaliar outros interferentes na estabilidade da vitamina C.

Palavras-chave: capacidade antioxidante, laticínios, Voltametria cíclica

\section{ABSTRACT}

Ascorbic acid has aroused particular interest due to its antioxidant capacity, which can prevent oxidative reactions. Cyclic voltammetry stands out as one of the main electrochemical techniques for antioxidant evaluation. The cheese and ricotta whey are subproducts of the dairy, and have several components that allow its use in various foods. The enrichment of subproducts with essential substances in food may make its use even more attractive by industries. Therefore, the aim of this study was to evaluate the stability of ascorbic acid in cheese and ricotta whey using cyclic voltammetry. For this, the cheese and ricotta whey were collected from a dairy industry of the Vale do Taquari-RS and were fortified with ascorbic acid and its stability was evaluated according to the electrochemical profiles of the samples. With the data obtained, it was concluded that one of the factors affecting directly the stability of ascorbic acid was the $\mathrm{pH}$, but more studies are needed to evaluate other interfering stability of vitamin C.

Keywords: antioxidant capacity, dairy, cyclic voltammetry. 


\section{INTRODUÇÃO}

A qualidade nutricional dos alimentos tem sido um dos fatores que mais preocupa os consumidores, e as indústrias de alimentos. O ácido ascórbico (AA), também conhecido como vitamina $C$, tem despertado um interesse em particular em função da sua contribuição em inúmeras atividades fisiológicas e sua eficiência em quantidades mínimas, sendo considerado essencial no funcionamento e manutenção de uma vida saudável (MANELA-AZULAY et al., 2003; 2 Z ZHANG e HAMAUZU, 2004). Estudos recentes nesta área vem sendo realizados, visando avaliar a influência dos processamentos e das tecnologias de armazenamento na composição química dos alimentos (HOWARD et al., 1999).

Outra importante característica do ácido ascórbico é sua capacidade antioxidante, que pode prevenir reações de oxidação e escurecimento dos alimentos, razão pela qual é utilizado em aromatizantes, na cura de carnes, na produção de pão, entre outros (PRÉSTAMO et al., 1993; LU e SEIB, 1998). No Brasil, a ingestão diária recomendada é de $60 \mathrm{mg}$, valor que pode facilmente ser alcançado pelo consumo de frutas e vegetais frescos (ANVISA, 2002).

O soro de queijo é um subproduto da indústria de laticínios gerado a partir da produção de queijo, podendo representar até $90 \%$ do volume de leite empregado, e com aproximadamente $55 \%$ dos nutrientes do leite. A utilização do soro de queijo na fabricação de outros alimentos vem sendo a melhor alternativa para agregar valor, e reduzir o descarte inadequado desse subproduto, evitando que funcione como agente de poluição ambiental (FLORENTINO, 2006; SANTOS e FERREIRA, 2001; SISO, 1996).

Uma das aplicações industriais do soro de queijo é na fabricação da ricota. Entretanto, nesse processo há geração de um novo subproduto, o soro de ricota. Este soro atualmente não é utilizado pelas indústrias, mas ainda contém diversos componentes que permitem sua utilização. O soro de ricota contém de 4 a $5 \%$ de lactose, e para degradação desse açúcar é preciso grande quantidade de oxigênio, o que representa um grande impacto ambiental quando descartado incorretamente. Sua elevada demanda bioquímica de oxigênio (DBO), na ordem de 30.000 a $40.000 \mathrm{mg}$ de oxigênio/litro de soro, pode promover a destruição da fauna e flora, o que faz com que alternativas para utilização deste subproduto sejam ainda mais importantes (MING, 2000; PENNA et al., 2009).

A utilização dos soros de queijo e de ricota no desenvolvimento de novos produtos vem sendo fruto de novos estudos (SANTOS e FERREIRA, 2001; PENNA et al., 2009). O enriquecimento desses subprodutos com substâncias essenciais na alimentação humana pode tornar seu uso ainda mais atrativo pelas indústrias.

A voltametria cíclica (VC) é uma técnica eletroquímica conveniente para o estudo de reações redox de espécies eletroativas em função da variedade de informações que podem ser obtidas, o que faz com que seja uma das técnicas eletroquímicas mais versáteis e amplamente utilizada para análises qualitativas $e$ quantitativas. Na VC, mede-se a resposta de corrente de um eletrodo a um ciclo linear de potenciais crescentes e decrescentes, sendo que a magnitude da corrente medida é proporcional à concentração da espécie eletroativa em solução (SCHOLZ, 2010).

Assim, se torna interessante avaliar a estabilidade do ácido ascórbico para possibilitar novas tecnologias e futuras aplicações destes soros na indústria alimentícia. Dessa forma o objetivo deste trabalho foi avaliar a estabilidade do ácido ascórbico em soro de queijo e soro de ricota através da técnica de voltametria cíclica.

\section{MATERIAL E MÉTODOS}

\subsection{Enriquecimento dos Soros}

Todos reagentes utilizados neste trabalho foram de grau analítico. O soro de queijo do tipo lanche e o soro de ricota oriundo da fabricação da ricota de soro de queijo lanche utilizados neste trabalho foram obtidos de uma indústria de laticínios do Vale do Taquari - RS. Ambos os soros foram enriquecidos a partir de uma solução aquosa de ácido ascórbico, de modo que sua concentração final nos soros fosse $1 \mathrm{mM}$. Todas as soluções foram armazenadas a $4^{\circ} \mathrm{C}$ durante o período de avaliação.

\subsection{Ensaios Eletroquímicos}

Os ensaios eletroquímicos foram 
realizados pela técnica de voltametria cíclica, em um potenciostato/galvanostato PGSTAT128N (AUTOLAB). Todas as medidas foram realizadas utilizando-se um eletrodo de carbono vítreo (GCE, $\mathrm{d}=3 \mathrm{~mm}$ ) como eletrodo de trabalho, um fio de platina como contra-eletrodo e um eletrodo de referência $\mathrm{Ag} / \mathrm{AgCl} 3 \mathrm{M}$. A obtenção dos voltamogramas cíclicos das amostras e do branco foi realizada pela aplicação de 1 ciclo na faixa de potenciais de 0,0 a $+0,6 \mathrm{~V}$, a uma velocidade de varredura de $20 \mathrm{mV} \mathrm{s}-1$. Todos os experimentos foram realizados à temperatura ambiente e após a purga do sistema com nitrogênio por 5 minutos.

Antes de cada medida, o eletrodo foi polido em alumina $0,5 \mu \mathrm{m}$ até a obtenção de uma superfície espelhada. Em seguida, foi lavado com etanol para a remoção de possíveis resíduos de alumina que possam ter ficado na superfície do GCE e sonicado por 5 minutos numa mistura de água:etanol (1:1). Posteriormente, o eletrodo foi lavado com etanol e, em sequência, com água ultrapura em abundância.

\subsection{Avaliação da Estabilidade do Ácido Ascórbico}

A estabilidade do ácido ascórbico foi avaliada por um período de 11 dias ou até que atingisse a degradação total. Para esta avaliação, foi feita a análise dos voltamogramas cíclicos obtidos, através da medida da carga sobre o pico de oxidação das amostras, entre o intervalo de 0,0 a +0,6 V em função do tempo de armazenamento das mesmas. Para fins de comparação, os mesmos testes foram realizados em solução tampão fosfato $0,1 \mathrm{~mol} \mathrm{L-1}(\mathrm{pH}=$ $4,5)$.

A quantidade de ácido ascórbico remanescente em solução foi medida através da razão Qt/Q0, onde Qt e Q0 são as cargas medidas sobre o pico de oxidação no tempo t e no tempo zero, respectivamente.

\section{RESULTADOS E DISCUSSÃO}

O ácido ascórbico foi facilmente dissolvido em água, e após a adição deste nos soros, a solução final se manteve homogênea. Testes preliminares em solução tampão fosfato demonstraram que uma solução de ácido ascórbico $1 \mathrm{mM}$ apresentava um pico suficientemente bem definido para sequência dos experimentos (Figura 1).
A estabilidade do ácido ascórbico em solução tampão pode ser vista na Figura 1. Nesta figura, os voltamogramas cíclicos da quantidade de ácido ascórbico remanescente em solução em função do tempo de armazenamento são mostrados. Como pode ser visto, o sinal eletroquímico do ácido ascórbico residual decresce com o passar dos dias, sendo que observa-se uma redução de mais de $70 \%$ do sinal analítico após três dias de armazenamento. De acordo com Fennema (2000), a vitamina C pode ser $100 \%$ degradada durante o preparo e armazenamento dos alimentos pela ação da luz, temperatura, $\mathrm{pH}$ elevado, íons metálicos como o $\mathrm{Cu}^{2+}$ e $\mathrm{Fe}^{+3}$, espécies reativas do oxigênio, umidade, entre outros.

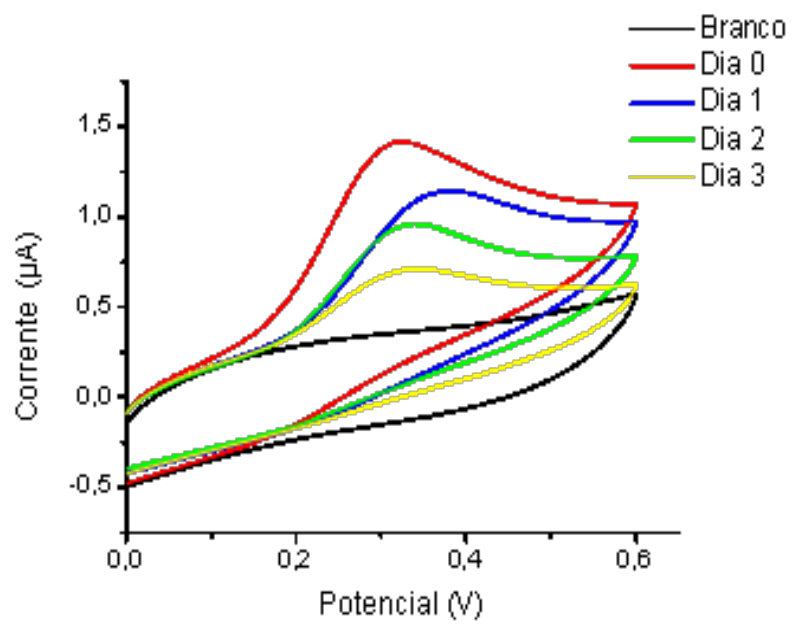

Figura 1. Voltamogramas cíclicos da quantidade de ácido ascórbico em solução tampão em função do tempo de armazenamento. Condições experimentais: ver seção 2.2.

O ácido ascórbico é facilmente oxidado sobre um eletrodo de carbono vítreo, o que constitui a base de sua determinação eletroquímica. Ele apresenta apenas um pico de oxidação devido à formação de um par redox irreversível com o ácido dehidroascórbico (PISOSCHI et al., 2014), cuja altura depende da concentração de ácido ascórbico presente em solução. Entretanto, dependendo das condições de armazenamento em função do tempo, o ácido ascórbico pode se degradar e a perda do sinal eletroquímico do AA presente em solução está relacionada à sua degradação.

A fim de avaliar a estabilidade do ácido ascórbico em soro de ricota e soro de queijo através da técnica de voltametria cíclica, o sinal 
eletroquímico do AA em solução foi estudado em função do tempo (Figura 2). Sabe-se que a diminuição do sinal eletroquímico do AA tende a cair em função do tempo. Assim, quanto menor a diminuição do sinal analítico, maior será a estabilidade do ácido ascórbico em solução. É possível observar que para ambos os soros, o comportamento da degradação da vitamina $C$ foi semelhante, com redução destes teores já após um dia de armazenamento. Porém, percebe-se que no soro de queijo esta redução se dá de forma menos acentuada. As equações de reta obtidas, $y=-0,0469 x+0,8928$ e $y=-0,0739 x$ $+0,8933$, para o soro de queijo e o soro de ricota, respectivamente, indicam que a exaustão de $A A$ nas soluções se dá em 19 dias para o soro de queijo e 12 dias para o soro de ricota, evidenciando a maior estabilidade do $A A$ na primeira solução. Um dos possíveis motivos para esta diferença deve-se ao $\mathrm{pH}$ de cada solução. Enquanto o soro de ricota apresenta um $\mathrm{pH}$ de 5,10 , o soro de queijo possui $\mathrm{pH}$ de 6,33.

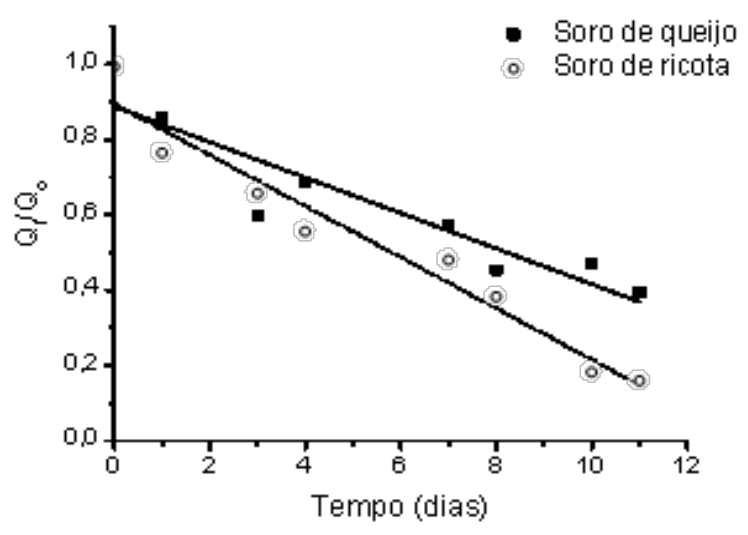

Figura 2. Variação do sinal analítico observado (Qt/Q0) em função do tempo de armazenamento.

Nogueira (2011), monitorou a influência do $\mathrm{pH}$ em diversos sucos de frutas, e verificou que após duas horas de armazenamento a quantidade de vitamina $C$ degradada variou entre $0,05 \mathrm{mg} / 100 \mathrm{~mL}$ a $1,2 \mathrm{mg} / 100 \mathrm{~mL}$ de suco. Danielli et al. (2009) acompanharam a degradação de vitamina $C$ no suco de laranja por 14 horas e encontraram uma porcentagem de degradação de 4,6\%. Branco et al. (2007) avaliaram a estabilidade deste antioxidante em uma mistura de suco de laranja com cenoura e detectaram perdas de ácido ascórbico de apenas $9,1 \%$ após 60 dias de armazenamento. Nogueira
(2011) verificou que as maiores porcentagens de degradação total da vitamina C ocorreram em sucos que possuíam menores teores iniciais desta vitamina. Atribuindo esse comportamento à presença de substâncias oxidantes, pois todos os sucos que os continham, a porcentagem de degradação total da vitamina $\mathrm{C}$ foi superior a $70 \%$.

\section{CONCLUSÕES}

Com os dados obtidos, concluiu-se que um dos fatores que afetaram de forma direta na estabilidade do ácido ascórbico foi o $\mathrm{pH}$, porém, mais estudos são necessários para avaliar outros interferentes na estabilidade da vitamina C.

\section{REFERÊNCIAS:}

1. Agência Nacional De Vigilância Sanitária (ANVISA); Resolução RDC $n^{\circ} 259$, de 20 de setembro de 2002. Regulamento técnico para rotulagem nutricional de alimentos embalados. República Federativa do Brasil, Brasília, DF, de 20 de setembro de 2002 [acesso em jan 2016]. Disponível em: <hhttp://www.ibravin.org.br/downloads/RDC $259 \% 20$ de $\% 2020 \% 20$ de $\% 20$ setembro\%20de $\% 202002$.pdf>

2. Branco IG, Sanjinez-Argandona EJ, Silva MM, Paula TM. Avaliação sensorial e estabilidade físico-química de um blend de laranja e cenoura. Ciência e Tecnologia de Alimentos. $2007 \quad$ Mar;27(1):7-12, doi:10.1590/S0101-20612007000100002.

3. Danieli F, Costa LRLG, Silva LC, Hara ASS, Silva AA. Determinação de vitamina $C$ em amostras de suco de laranja in natura e amostras comerciais de suco de laranja pasteurizado e envasado em embalagem Tetra Pak. Rev Inst Ciênc Saúde. 2009 Out/Dez;27(4):361-365.

4. Fennema OR. Química de los Alimentos. Zaragoza: Acribia; 2000. 1258 p.

5. Florentino ER. Aproveitamento do soro de queijo de coagulação enzimática [dissertação]. Natal (RN): Universidade Federal do Rio Grande do Norte; 2006. 150p.

6. Howard LA, Wong AD, Perry AK, Klein BP. $\beta$ carotene and ascorbic acid retention in fresh and processed vegetables. Journal of Food Science. $1999 \quad$ Sept;64(5):929-936, doi:10.1111/j.1365-2621.1999.tb15943.x.

7. Lu X, Seib PA. Assay of dehydroascorbic acid 
in bread and dough added as a crystalline dimer. Cereal Chemistry. 1998 Mar;75(2):200-206, doi:10.1094/CCHEM.1998.75.2.200.

8. Manela-Azulay M, Lacerda CAM, Perez MA, Filgueira AL, Cuzzi T. Vitamin C. Anais Brasileiros de Dermatologia; 2003; 78(3); Rio de Janeiro.

9. Ming P. Propriedades nutricionais das proteínas de soro de leite. Revista leite e derivados. 2000; 9(52):64-68.

10. Nogueira FS. Teores de Ácido L-Ascórbico em frutas e sua estabilidade em sucos [dissertação]. Campos dos Goytacazes (RJ): Universidade Estadual do Norte Fluminense Darcy Ribeiro; 2011.

11. Penna ALB, Almeida KE, Oliveira MN. Soro de leite: importância biológica, comercial e industrial - principais produtos. In: Oliveira $\mathrm{MN}$, editor. Tecnologia de produtos lácteos funcionais; 2009, São Paulo: Atheneu, p. 251-276.

12. Pisoschi AM, Pop A, Serban Al, Fafaneata C. Electrochemical methods for ascorbic acid determination. Electrochimica Acta. 2014
Mar;121:443-460, doi:10.1016/j.electacta.2013.12.127.

13. Préstamo G, Manzano P. Peroxidases of selected fruits and vegetables and the possible use of ascorbic acid as an antioxidant. HortScience. 1993 Jan;28(1):4850.

14. Santos JPV, Ferreira CLLF. Alternativas para o aproveitamento de soro de queijo nos pequenos e médios laticínios. Revista do Instituto de Laticínios Cândido Tostes. 2001; 56(321):44-50.

15. Scholz F. Electroanalytical Methods: Guide to Experiments and Applications. Berlin Heidelberg Germany. Springer. 2010. 57-106 p, doi: 10.1007/978-3-642-02915-8.

16. Siso MIG. The biological utilization of cheese whey: a review. Bioresource Technology. 1996; 57:1-11.

17. Zhang D, Hamauzu Y. Phenolics, ascorbic acid, carrotenoids and antioxidant activity of broccoli and their changes during conventional and microwave cooking. Food Chemistry. 2004 Dec;88(4):503-509, doi:10.1016/j.foodchem.2004.01.065 